Pacific Journal of Mathematic 


\title{
THE RELATIONS BETWEEN A SPECTRAL \\ OPERATOR AND ITS SCALAR PART
}

\author{
S. R. FOGUEL
}

1. Introduction. It is shown in Dunford's theory of spectral operators, that every spectral operator $T$ can be decomposed into the sum of a scalar operator $S$, and a generalized nilpotent $N$ [1]. We study here properties which are inherited by $S$ from $T$. The main results are:

1. If the spectral operator $T$ is compact, weakly compact, or has a closed range, then respectively $S$ is compact, weakly compact, or has a closed range.

2. The relations between the point spectra, continuous spectra, and residual spectra of $S$ and $T$ are investigated.

3. If the sum of two commuting spectral operators is spectral, then the sum of their scalar parts is scalar.

2. Notation. Most of the notation is taken from [1]. Let $X$ be a complex Banach space. A spectral measure is a set function $E(\cdot)$, defined on Borel sets in the complex plane, whose values are projections on $X$, which satisfy :

$(\alpha)$ For any two Borel sets $\sigma$ and $\delta E(\sigma) E(\delta)=E(\sigma \cap \delta)$.

$(\beta)$ Let $\Phi$ be the void set and $p$ the complex plane.

Then

$$
E(\Phi)=0 \text { and } E(p)=I
$$

( $\gamma$ ) There exists a constant $M$ such that $\mid E(\sigma) ! \leqq M$, for every Borel set $\sigma$.

( $\delta$ ) The vector valued set function $E(\cdot) x$ is countable additive for each $x \in X$.

The operator $T$ is a spectral operator, whose resolution of the identity is the spectral measure $E(\cdot)$ if

(a) for every Borel set $\sigma E(\sigma) T=T E(\sigma)$.

(b) Let $T_{\alpha}$ denote the restriction of $T$ to the subspace $E(\alpha) X, \quad\left(T_{\alpha}\right.$ $=T \mid E(\alpha) X)$ then

Received March 13, 1957, in revised form August 19, 1957. This paper is a part of a dissertation presented for the degree of Doctor of Philosophy in Yale University. The author wishes to express his gratitude to Professor N. Dunford for his guidance and kind encouragement. 


$$
\sigma\left(T_{\alpha}\right) \subset \bar{\alpha}
$$

where $\sigma(A)$ is the spectrum of $A$.

Throughout the paper $T$ denotes a spectral operator, $E(\cdot)$ its resolution of the identity, $S$ its scalar part given by $S=\int_{p} \lambda E(d \lambda), N$ its radical given by $N=T-S$. The operator $N$ is a generalized nilpotent, and the operators $N, S, T, E(\alpha)$ commute [1]. A spectral operator is of finite type, if for some integer $n, N^{n+1}=0$. We shall denote $N \cdot E(\langle 0\rangle)$ by $N_{0}$, hence $N_{0}=T E(\langle 0\rangle)=E(\langle 0\rangle) T$.

3. Topological properties. In this section, several topological properties will be shown to be valid for $S$ whenever they are valid for $T$. The following lemma will be used.

LEMMA $1 . S$ is in the uniformly closed operator algebra generated by the projections $E(\alpha)$ with $0 \notin \bar{\alpha}$.

Proof. $S=\int_{\sigma(T)} \lambda E(d \lambda)$ and $\sigma(T)$ is bounded, see [1] Theorem 1. Given $\varepsilon>0$ let $\sigma(T)$ be divided into the disjoint sets $\alpha_{0}, \alpha_{1}, \cdots, \alpha_{n}$ with

$$
\begin{array}{ll}
0 \in \alpha_{0}, \quad 0 \notin \bar{\alpha}_{i}, & i=1,2, \cdots, n \text { and } \\
\operatorname{diam}\left(\alpha_{i}\right)<\varepsilon & i=0,1,2, \cdots, n .
\end{array}
$$

Let $\lambda_{0}=0$ and $\lambda_{i} \in \alpha_{i}$. Then

$$
\left|S-\sum_{i=1}^{n} \lambda_{i} E\left(\alpha_{i}\right)\right|=\left|\int_{\sigma(T)}\left(\lambda-\sum_{i=0}^{n} \lambda_{i} \chi_{\alpha_{i}}(\lambda)\right) E(d \lambda)\right| .
$$

If $\lambda \in \sigma(T)$ then

$$
\left|\lambda-\sum_{i=0}^{n} \lambda_{i} \chi_{\alpha_{i}}(\lambda)\right| \leqq \varepsilon
$$

Now by [1], p. 330, for every bounded measurable function defined on $\sigma(T)$

$$
\left|\int_{\sigma(T)} f(\lambda) E(d \lambda)\right| \leqq \sup \{|f(\lambda)|, \quad \lambda \in \sigma(T)\} \cdot 4 M .
$$

Hence

$$
\left|S-\sum_{i=1}^{n} \lambda_{i} E\left(\alpha_{i}\right)\right| \leqq 4 M \varepsilon
$$

THEOREM 1. Let $\mathfrak{A}$ be a uniformly closed right (left) ideal in the algebra of operators on $X$. If $T$ belongs to $\mathfrak{A}$ so do $S, N$, and $E(\alpha)$ with $0 \notin \bar{\alpha}$.

Proof. By condition b of $\S 2 T_{\alpha}$ with $0 \notin \bar{\alpha}$ possesses a bounded 
everywhere defined inverse $T_{\alpha}^{-1}$. Let us define $P_{\alpha}$ by $P_{\alpha} x=T_{\alpha}^{-1} E(\alpha) x$, $x \in X, 0 \notin \bar{\alpha} . \quad P_{\alpha}$ is a bounded everywhere defined operator. Now

$$
T P_{\alpha} x=T\left(T_{\alpha}^{-1} E(\alpha) x\right)=\left(T T_{\alpha}^{-1}\right)(E(\alpha) x)=E(\alpha) x .
$$

Also

$$
P_{\alpha} T x=T_{\alpha}^{-1} E(\alpha) T x=T_{\alpha}^{-1} T E(\alpha) x=\left(T_{\alpha}^{-1} T\right) E(\alpha) x=E(\alpha) x .
$$

Hence if $0 \notin \bar{\alpha}$ then $E(\alpha) \in \mathfrak{A}$. Note that this fact remains true even if $\mathfrak{A}$ is not uniformly closed. Now by Lemma $1 S \in \mathfrak{A}$ and therefore $N \in \mathfrak{A}$ too.

Corollary 1. If $T$ is compact then so are $S, N$ and $E(\alpha)(0 \notin \bar{\alpha})$.

Corollary 2. If $T$ is weakly compact then so are $S, N$ and $E(\alpha)$ with $0 \notin \bar{\alpha}$.

Corollary 3. If $T X \subset Y$ where $Y$ is a closed subspace of $X$, then $S X \subset Y$ and $N X \subset Y$ and $E(\alpha) X \subset Y, 0 \notin \bar{\alpha}$. Hence

$$
S X \cup N X \cup \cup(E(\alpha) X \mid 0 \notin \bar{\alpha}) \subset \overline{T X}
$$

and if the range of $T$ is separable so are the ranges of $S, N$ and $E(\alpha)$, $0 \notin \bar{\alpha}$.

Corollary 4. If $A_{0} T=0\left(T A_{0}=0\right)$ then $A_{0} S=A_{0} N=0$ and $A_{0} E(\alpha)=0$, $0 \notin \bar{\alpha}\left(S A_{0}=N A_{0}=E(\alpha) A_{0}=0\right.$ if $\left.0 \notin \bar{\alpha}\right)$. In particular $T$ is a spectral operator of finite type if and only if some power of $N$ annihilates $T$.

Corollary 5. If $T x=0$ then $N x=S x=E(\alpha) x=0$ where $\bar{\alpha}$ does not contain 0 .

CoROLlaRy 6. If $\left(x_{n}\right)$ is a bounded sequence of vectors, and the sequence $\left(T x_{n}\right)$ has a limit then the sequences $\left(S x_{n}\right),\left(N x_{n}\right)$ and $\left(E(\alpha) x_{n}\right)$ with $0 \notin \bar{\alpha}$ have limits.

To prove these corollaries one has to note that:

(a) The classes of compact and weakly compact operators are uniformly closed two-sided ideals. (See [3] Chapter 6).

(b) The classes of operators $A$ satisfying $A X \subset Y$ or $A_{0} A=0$ are uniformly closed right ideals.

(c) The classes of operators $A$ satisfying $A x=0$ or $A A_{0}=0$ or the limit of $A x_{n}$ exists are uniformly closed left ideals.

REMark To CoRollary 6. By the proof of Theorem 1 the sequence $\left(E(\alpha) x_{n}\right), 0 \notin \bar{\alpha}$, has a limit whenever the sequence $\left(T x_{n}\right)$ has, even if the sequence $\left(x_{n}\right)$ is not bounded. 
Theorem 2. $A T=0$ if and only if $A E(p-\langle 0\rangle)=0 \quad(A=A E(\langle 0\rangle))$ and $A N_{\mathrm{v}}=0$. Similarly $T A=0$ if and only if $E(p-\langle 0\rangle) A=N_{0} A=0$.

Proof. If $A N_{0}=A E(p-\langle 0\rangle)=0$ then $A E(\alpha)=A E(p-\langle 0\rangle) E(\alpha)=0$ if $0 \notin \bar{\alpha}$, thus by Lemma $1 A S=0$. Now

$$
A N=A N E(\langle 0\rangle)+A N E(p-\langle 0\rangle)=A N_{0}+(A E(p-\langle 0\rangle)) N=0 .
$$

Thus $A T=A S+A N=0$. Conversely if $A T=0$ then $A N_{0}=A T E(\langle 0\rangle)=0$, and $A E(\alpha)=0$ if $0 \notin \bar{\alpha}$. Now for each $x \in X$

$$
A E(p-\langle 0\rangle) x=\lim A E\left\{z\left|\frac{1}{n} \leqq\right| z \mid\right\} x=0
$$

by countable additivity.

The second half of the theorem is proved in the same way.

Using Corollary 5 one can prove in the same way that $T x=0$ if and only if $N_{0} x=E(p-\langle 0\rangle) x=0$. $A=0$.

Corollary 1. If $E(\langle 0\rangle)=0$, then $A T=0$ or $T A=0$ if and only if

Proof. By Theorem 2 if $A T=0$ or $T A=0$ then $A=A E(\langle 0\rangle)$ or $A=E(\langle 0\rangle) A$.

Corollary 2. If $E(\langle 0\rangle)=0$ then $\overline{T X}=X$.

Proof. If $\overline{T X} \neq X$ then there exists a bounded functional $x^{*} \neq 0$ such that $x^{*}(T X)=0$. Let $A x=x^{*}(x) x_{1}$ where $x_{1}$ is any vector different from 0 . $A T=0$ and $A \neq 0$ which contradicts Corollary 1 .

Theorem 3. If $T$ has a closed range so does $S$.

1. Proof. Let $E(\langle 0\rangle)=0$ then Corollary 2 of Theorem 2 shows that $\overline{T X}=X$. But by assumption $\overline{T X}=T X$, thus $T X=X$. Also, the operator $T$ is one-to-one by [1] p. 327 and thus $T$ possesses a bounded everywhere defined inverse. Thus $0 \notin \sigma(S)=\sigma(T)$ and $S X=X$.

2. Let $E(\langle 0\rangle) \neq 0$. The operator $T_{p-\langle 0\rangle}$ is a spectral operator whose resolution of the identity $F(\cdot)$ is given by $F(\alpha)=E(\alpha) E(p-\langle 0\rangle)=E(\alpha$ $-\langle 0\rangle)$, hence $F(\langle 0\rangle)=0$. Now if $T_{p-\langle<\rangle} x_{n} \rightarrow y(y \in E(p-\langle 0\rangle) X)$, then, there exists a vector $x$ in $X$ such that $T x=y$, because $T$ has a closed range. Therefore

$$
T_{p-\langle 0\rangle}(E(p-\langle 0\rangle) x)=T E(p-\langle 0\rangle) x=E(p-\langle 0\rangle) T x=E(p-\langle 0\rangle) y=y .
$$


Hence $T_{p-<>>}$ satisfies the same conditions assumed for $T$ in the first part and therefore $0 \notin \sigma\left(T_{p-\langle<\rangle}\right)$ and

$$
S_{p-\langle 0\rangle} X=E(p-\langle 0\rangle) X \text {, but } S_{p-\langle 0\rangle} X=S X,
$$

so $S$ has a closed range.

By the proof of the last theorem it follows that if $T$ has a closed range then $0 \notin \sigma\left(T_{p-\langle 0\rangle}\right)$, hence 0 is an isolated point of the spectrum of $T$.

Theorem 4. The operator $T$ has a closed range if and only if

1. 0 is an isolated point of $\sigma(T)$.

2. The operator $N_{0}$ has a closed range.

Proof. We proved that Condition 1 is necessary. Now if $N_{0} x_{n} \rightarrow y$ then $E(\langle 0\rangle) N_{0} x_{n} \rightarrow E(\langle 0\rangle) y$ but $E(\langle 0\rangle) N_{0}=N_{0}$ thus $E(\langle 0\rangle) y=y$. Also $N_{0}$ $=T E(\langle 0\rangle)$ and $T$ has a closed range, thus if $T\left(E(\langle 0\rangle) x_{n}\right) \rightarrow y$ then for some $x, T x=y$. Hence $T E(\langle 0\rangle) x=N_{0} x=E(\langle 0\rangle) y=y$. Conversely if 1 . and 2. are satisfied let $T x_{n} \rightarrow y$. Then

$$
\begin{gathered}
T E(p-\langle 0\rangle) x_{n}+T E(\langle 0\rangle) x_{n}=T E(p-\langle 0\rangle) x_{n}+N_{0} x_{n} \\
\rightarrow y=E(p-\langle 0\rangle) y+E(\langle 0\rangle) y .
\end{gathered}
$$

Multiplying this equation by $E(p-\langle 0\rangle)$ and $E(\langle 0\rangle)$ one gets the following two equations

$$
\begin{aligned}
& T E(p-\langle 0\rangle) x_{n} \rightarrow E(p-\langle 0\rangle) y \\
& N_{0} x_{n} \rightarrow \quad E(\langle 0\rangle) y
\end{aligned}
$$

By 1. $T_{p-<0\rangle}$ possesses a bounded everywhere defined inverse. Hence, for some $x_{1}$ in $E(p-\langle 0\rangle) X, T x_{1}=E(p-\langle 0\rangle) y$.

By 2. for some vector $x_{2}, N_{0} x_{2}=E(\langle 0\rangle) y$. Thus

$$
T\left(x_{1}+E(\langle 0\rangle) x_{2}\right)=T x_{1}+N_{0} x_{2}=y .
$$

4. Properties of spectral points. Let $A$ be a bounded linear operator on $X$, define

$\sigma_{p}(A)=\{\lambda \mid \lambda I-A$ is not one-to-one $\}$

$\sigma_{c}(A)=\{\lambda \mid \lambda I-A$ is one-to-one and $(\lambda I-A) X$ is dense in $X$, but not equal to $X\}$.

$\sigma_{r}(A)=\{\lambda \mid \lambda I-A$ is one-to-one and $\overline{(\lambda I-A) X} \neq X\}$.

(See [6] p. 292.)

The sets $\sigma_{p}(A), \sigma_{c}(A)$ and $\sigma_{r}(A)$ are disjoint and

$$
\sigma(A)=\sigma_{p}(A) \cup \sigma_{c}(A) \cup \sigma_{r}(A) .
$$


THEOREM 1. If $T$ is a spectral operator of finite type, then $\lambda \in \sigma_{p}(T)$ if and only if $E(\langle\lambda\rangle) \neq 0$, and $\lambda \in \sigma_{c}(T)$ if and only if $E(\langle\lambda\rangle)=0$, and $\lambda \in \sigma(T)$. Thus $\sigma(T)=\sigma_{p}(T) \cup \sigma_{c}(T)$.

Proof. If $E(\langle\lambda\rangle) \neq 0$ let $x \in E(\langle\lambda\rangle) X, x \notin 0$, then

$$
S x=\int_{\sigma(T)} \mu E(d \mu) x=\int_{\sigma(T)} \mu E(d \mu) E(\langle\lambda\rangle) x=\lambda x .
$$

Let $\nu$ be the first integer such that $N^{\nu} x=0$, then

$$
T N^{\nu-1} x=S N^{\nu-1} x+N^{\nu} x=N^{\nu-1} S x=\lambda N^{\nu-1} x,
$$

therefore $\lambda \in \sigma_{p}(T)$. If $E(\langle\lambda\rangle)=0$ then Corollary 2 of Theorem 2, 33 , applied to $\lambda I-T$, shows that $\overline{(\lambda I-T) X}=X$. Also, by [1] Lemma $1, \lambda I-T$ is one-to-one and thus $\lambda \in \sigma_{c}(T)$.

Theorem 2. $\sigma_{c}(S) \subset \sigma_{c}(T)$ and $\sigma_{p}(T) \cup \sigma_{r}(T) \subset \sigma_{p}(S)$.

Proof. If $\lambda \in \sigma_{c}(S)$ then $E(\langle\lambda\rangle)=0$, and by the last part of the proof of Theorem 1, $\lambda \in \sigma_{c}(T)$. Thus $\sigma_{c}(S) \subset \sigma_{c}(T)$ and

$$
\sigma_{p}(T) \cup \sigma_{r}(T)=\sigma(T)-\sigma_{c}(T) \subset \sigma(T)-\sigma_{c}(S)=\sigma(S)-\sigma_{c}(S)=\sigma_{p}(S) .
$$

If $E(\langle\lambda\rangle)=0$ then $\lambda \in \sigma_{c}(T)$. Let us examine therefore the case where $E(\langle\lambda\rangle) \neq 0$. To simplify notation assume that $\lambda=0$.

Theorem 3. Let $E(\langle 0\rangle) \neq 0$ then

1. $0 \in \sigma_{p}(T)$ if $N_{0}$ is not one-to-one on $E(\langle 0\rangle) X$.

2. $0 \in \sigma_{c}(T)$ if $N_{0}$ is one-to-one on $E(\langle 0\rangle) X$ and $\overline{N_{0}(E(\langle 0\rangle) X)}=E(\langle 0\rangle) X$.

3. $0 \in \sigma_{r}(T)$ if $N_{0}$ is one-to-one on $E(\langle 0\rangle) X$ and $\overline{N_{0}(E(\langle 0\rangle) X} \neq E(\langle 0\rangle) X$.

Proof.

1. If there exists a vector $x$ such that $x \neq 0, x=E(\langle 0\rangle) x$ and $N_{0} x=0$ then

$$
T x=T E(\langle 0\rangle) x=N_{0} x=0
$$

2. The operator $T_{p-<\rangle\rangle}$ is one-to-one on $E(p-\langle 0\rangle) X$ by [1] Lemma 1. Now if $N_{0}$ is one-to-one on $E(\langle 0\rangle) X$ then $T$ is one-to-one on $X$ : If $T x=0$ then $E(\langle 0\rangle) T x=N_{0} x=N_{0} E(\langle 0\rangle) x=0$ and $T E(p-\langle 0\rangle) x=T_{p-<0} E(p$ $-\langle 0\rangle) x=0$. Thus $E(\langle 0\rangle) x=0$ and $E(p-\langle 0\rangle) x=0$, but then $x=E(\langle 0\rangle) x$ $+E(p-\langle 0\rangle) x=0$. Now by Corollary 2 of Theorem 2 , $\S 3$

$$
\overline{T_{p-<0} E(p-\langle 0\rangle) X}=E(p-\langle 0\rangle) X
$$


and by assumption

$$
\overline{N_{0} X}=E(\langle 0\rangle) X
$$

but

$$
\overline{T X} \supset \overline{T_{p-<0} E(p-\langle 0\rangle) X}
$$

and

$$
\overline{T X} \supset \overline{N_{0} X}
$$

therefore

$$
\overline{T X} \supset X \text {. }
$$

3. By Part 2, $T$ is one-to-one. Let $x$ be a vector in $E(\langle 0\rangle) X$ whose distance from $N_{0} X$ is greater than some positive number $r$. Let $y$ be any vector in $X$. Then

$$
|x-T y|=\left|x-N_{0} y-T E(p-\langle 0\rangle) y\right| \text {. }
$$

Hence

$$
\begin{aligned}
|x-T y| & \geqq \frac{1}{M}\left|E(\langle 0\rangle)\left[x-N_{0} y-T E(p-\langle 0\rangle) y\right]\right| \\
& =\frac{1}{M}\left|x-N_{0} E(\langle 0\rangle) y\right| \geqq \frac{r}{M} .
\end{aligned}
$$

Hence

$$
x \notin \overline{T X} .
$$

The next theorem is valid for separable spaces only.

THEOREM 4. If $X$ is separable, then $\sigma_{p}(T) \cup \sigma_{r}(T)$ is countable.

Proof. Theorems 1 and 2 show that $\sigma_{p}(T) \cup \sigma_{r}(T) \subset \sigma_{p}(S)=\{\lambda \mid E(\langle\lambda\rangle)$ $\neq 0\}$. For any $\lambda$ in $\sigma_{p}(S)$ let $x_{\lambda}$ be a vector satisfying $\left|x_{\lambda}\right|=1$ and $E(\langle\lambda\rangle) x_{\lambda}=x_{\lambda}$. Now if $\lambda_{1} \neq \lambda_{2}$ then

$$
\left|x_{\lambda_{1}}-x_{\lambda_{2}}\right| \geqq \frac{1}{M}\left|E\left(\left\langle\lambda_{1}\right\rangle\right)\left(x_{\lambda_{1}}-x_{\lambda_{2}}\right)\right|=\frac{\left|x_{\lambda_{1}}\right|}{M}=\frac{1}{M} .
$$

The set $\left\{x_{\lambda} \mid \lambda \in \sigma_{p}(S)\right\}$ is separable because $X$ is, hence the set is countable.

We conclude this discussion by studying another subset of the spectrum.

Definition. Let $A$ be a bounded linear operator on $X$, then $\sigma_{0}(A)$ 
$=\left\{\lambda \mid\right.$ there exists a sequence $\left(x_{n}\right)$ such that $\left|x_{n}\right|=1$ and $\left.(\lambda I-A) x_{n} \rightarrow 0\right\}$. See [5] p. 51.

LemMa 1. $\sigma_{p}(S) \subset \sigma_{0}(T)$.

Proof. Let $x \neq 0$ satisfy $S x=\lambda x$. If for some $n, N^{n} x=0$, let us take the first such integer. Then

$$
T N^{n-1} x=(S+N) N^{n-1} x=N^{n-1} S x=\lambda N^{n-1} x,
$$

and thus $\lambda \in \sigma_{p}(T) \subset \sigma_{0}(T)$. If for every $n, N^{n} x \neq 0$ then

$$
T \frac{\left(N^{n} x\right)}{\left|N^{n} x\right|}=(S+N) \frac{N^{n} x}{\left|N^{n} x\right|}=\lambda \frac{N^{n} x}{\left|N^{n} x\right|}+\frac{N^{n+1} x}{\left|N^{n} x\right|} .
$$

It is enough to show that for some subsequence $n_{i}$

$$
\frac{\left|N^{n_{i}+1} x\right|}{\left|N^{n_{i}} x\right|} \rightarrow 0
$$

Let us assume, to the contrary, that for some $\varepsilon>0\left|N^{n+1} x\right| \geqq \varepsilon\left|N^{n} x\right|$ for all $n$, then

$$
|x| \leqq \frac{|N x|}{\varepsilon} \leqq \frac{\left|N^{2} x\right|}{\varepsilon^{2}} \leqq \cdots \leqq \frac{\left|N^{n} x\right|}{\varepsilon^{n}}
$$

but this would imply that

$$
\begin{aligned}
\lim _{n \rightarrow \infty} \sqrt[n]{\left|N^{n}\right|}=\lim _{n \rightarrow \infty} \sqrt[n]{\left|N^{n}\right| \sqrt[n]{|x|}} \geqq \lim _{n \rightarrow \infty} \sup \sqrt[n]{\left|N^{n} x\right|} \\
\geqq \lim _{n \rightarrow \infty} \sup \varepsilon \sqrt[n]{|x|}=\varepsilon .
\end{aligned}
$$

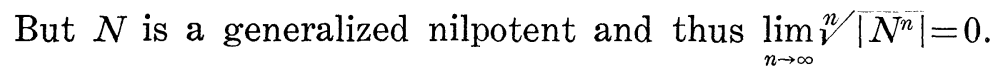

THEOREM 5. $\sigma(T)=\sigma_{0}(T)$.

Proof. By Theorem 2 and Lemma $1 \sigma_{p}(T) \cup \sigma_{r}(T) \subset \sigma_{p}(S) \subset \sigma_{0}(T)$. Thus it is enough to show that $\sigma_{c}(T) \subset \sigma_{0}(T)$. Let $\lambda \in \sigma_{c}(T)$ we may assume that $\lambda=0$. If $0 \notin \sigma_{0}(T)$ then $|T x| \geqq \varepsilon|x|, x \in X$, for some positive $\varepsilon$. This implies that $T X$ has a closed range, but $\overline{T X}=X$ hence $T X=X$, which contradicts the assumption that $0 \in \sigma_{c}(T)$.

Let us conclude this section with a few examples.

1. Define in $l_{1}$ the generalized nilpotent operator $N$ by

$$
N\left(x_{1}, x_{2}, x_{3}, \cdots\right)=\left(x_{2}, 0, x_{4}, 0, \cdots\right)
$$

and let $S=0 . \quad S$ is compact while $T$ is not weakly compact.

2. Let $X$ be the space of continuous functions on $[0,1]$ vanishing 
at the point 0 . Define $N$ by $N f=g, g(x)=\int_{0}^{x} f(s) d s$, and let $S=0 . \quad S$ has a closed range while $T$ does not. $0 \in \sigma_{p}(S)$ but $0 \in \sigma_{c}(T)$.

3. Let $N$ be defined as in 2, and $S=I . T$ and $S$ have closed ranges but the range of $N$ is not closed.

5. Decompositions of spectral operators. Let $T_{1}, \cdots, T_{n}$ be $n$ commuting operators. There exists a minimal algebra of operators $\mathfrak{A}$, with the properties:

1. $T_{i} \in \mathfrak{A}, i=1,2, \cdots, n$.

2. If $U \in \mathfrak{A}$ and $U^{-1}$ is a bounded everywhere defined operator then $U^{-1} \in \mathfrak{A}$.

3. The algebra $\mathfrak{A}$ is uniformly closed.

This algebra will be called the full algebra generated by $T_{1}, \cdots, T_{n}$, and it is a commutativealgebra. Let $\Delta_{\mathfrak{A}}$ denote the space of homomorphisms from $\mathfrak{U}$ to the algebra of complex numbers. By Condition 2, and the Gelfand theory [4], if $U \in \mathfrak{A}$ then $\sigma(U)=\left\{\mu(U) \mid \mu \in \Delta_{\mathscr{U}}\right\}$; thus if $\mu(U)=0$ for each $\mu \in \Delta_{\mathfrak{M}}$ then $U$ is a generalized nilpotent.

Lemma 1. Every scalar operator $S$ is the sum $S_{1}+i S_{2}$ where $S_{1}$ and $S_{2}$ are scalar operators and

1. $S_{1} S_{2}=S_{2} S_{1}$.

2. $\sigma\left(S_{1}\right)$ and $\sigma\left(S_{2}\right)$ are sets of real numbers.

3. The Boolean algebra of projections generated by the resolutions of the identity of $S_{1}$ and $S_{2}$ is bounded.

Proof. Let $E(\cdot)$ be the resolution of the identity of $S$; then

$$
\begin{aligned}
S=\int z E(d z) & =\int(x+i y) E(d z)=\int x E(d z)+i \int y E(d z) \\
& =\int \lambda E_{1}(d \lambda)+i \int \lambda E_{2}(d \lambda)
\end{aligned}
$$

where

$$
\begin{aligned}
& E_{1}(\alpha)=E\{z \mid z=x+i y \text { and } x \in \alpha\} \\
& E_{2}(\alpha)=E\{z \mid z=x+i y \text { and } y \in \alpha\}
\end{aligned}
$$

Conditions 1,2 , and 3 are readily verified.

THEOREm 1. Let $T$ be a spectral operator. Then there exist two operators $R$ and $J$ such that

1. $T=R+i J$ and $R J=J R$

2. The sets $\sigma(R)$ and $\sigma(J)$ are real sets.

3. $R$ is a scalar operator and $J$ is a spectral operator. 
4. The Boolean algebra of projections generated by the resolutions of the identity of $R$ and $J$ is bounded.

If $R_{1}$ and $J_{1}$ satisfy Conditions 1 and 2 , then they are spectral operators and there exists a generalized nilpotent $M$ such that

$$
R_{1}=R+M, \quad J_{1}=J+i M .
$$

REMark. By the last assertion and Theorem 8 of [1] Conditions 1, 2 , and 3 insure uniqueness. We shall call $R$ the real part of $T$ and $J$ the imaginary part of $T$.

Proof. Let $T=S+N$. Using the notation of Lemma 1, put $R=S_{1}$, $J=S_{2}-i N$, and Conditions 1., 2., 3., and 4. follow by Lemma 1. Now, if $R_{1}$ and $J_{1}$ satisfy 1 ,, and 2., then by Theorem 5 of [1], the operators $R, J, R_{1}, J_{1}$ commute. Let $\mathfrak{A}$ be the full algebra generated by these operators, if $\mu \in \Delta_{\mathfrak{A}}$ then

$$
0=\mu(T-T)=\mu\left(R-R_{1}\right)+i \mu\left(J-J_{1}\right)
$$

but $\mu\left(R-R_{1}\right)$ and $\mu\left(J-J_{1}\right)$ are real numbers by Condition 2 . Hence

$$
\mu\left(R-R_{1}\right)=\mu\left(J-J_{1}\right)=0 .
$$

Thus if $M=R-R_{1}$ then $M$ is a generalized nilpotent and $J-J_{1}=i M$.

LEMMA 2. Every scalar operator $S$ can be written as the product of two scalar operators $T_{1}$ and $T_{2}$ which satisfy

1. $T_{1} T_{2}=T_{2} T_{1}=S$.

2. $\sigma\left(T_{1}\right)$ is a set of non-negative numbers and $\sigma\left(T_{2}\right)$ is a subset of the unit circle.

3. The Boolean algebra of projections generated by the resolutions of the identity of $T_{1}$ and $T_{2}$ is bounded.

Proof. It follows from the multiplicative property of the spectral measure $E(\cdot)$ of $S$ that

$$
S=\int \lambda E(d \lambda)=\int|\lambda| E(d \lambda) \int \operatorname{sgn} \lambda E(d \lambda) .
$$

Thus $S=T_{1} T_{2}$ where

$$
T_{1}=\int|\lambda| E(d \lambda)=\int \mu E_{1}(d \mu) \text { if } E_{1}(\cdot)
$$

is defined by

$$
E_{1}(\alpha)=E\{\lambda|| \lambda \mid \in \alpha\}
$$




$$
T_{2}=\int \operatorname{sgn} \lambda E(d \lambda)=\int \mu E_{2}(d \mu)
$$

where

$$
E_{2}(\alpha)=E\{\lambda \mid \operatorname{sgn} \lambda \in \alpha\} .
$$

It is easy to verify Conditions 1,2 , and 3 .

THEOREM 2. Let $T$ be a spectral operator. Then there exist two operators $P$ and $U$ such that

1. $T=P U=U P$.

2. $\sigma(P)$ is a set of non-negative numbers and $\sigma(U)$ is a subset of the unit circle.

3. $U$ is a scalar operator and $P$ is spectral.

4. The Boolean algebra of projections generated by the resolutions of the identity of $P$ and $U$ is bounded.

If $P_{1}$ and $U_{1}$ satisfy 1 . and 2. , then they are spectral operators and $U_{1}=U+N_{1} P_{1}=P+N_{2}$ where $N_{1}$ ond $N_{2}$ are generalized nilpotents and

$$
N_{2}=\sum_{n=0}^{\infty}\left(-N_{1} U^{-1}\right)^{n+1} P .
$$

REMARK. By the last assertion Conditions 1, 2, and 3 insure uniqueness. The operator $P$ will be called the absolute value of $T$ and $U$ the argument of $T$.

Proof. Let $T=S+N$. Using the notation of Lemma 2 put $P=\left(T_{1}\right.$ $+T_{2}^{-1} N$ ) and $U=T_{2}$, then $P U=T$ because $T_{2} N=N T_{2}$ (Theorem 8 of [1]). Now, Conditions $1,2,3$, and 4 follow by Lemma 2 . Let $P_{1}$ and $U_{1}$ satisfy 1 and 2 ; then by Theorem 8 of $[1], P_{1}, U_{1}, P, U$ commute. Let $\mathfrak{A}$ be the full algebra generated by these operators. If $\mu \in \Delta_{\mathfrak{A}}$ then $\mu(T)=\mu(P) \mu(U)=\mu\left(P_{1}\right) \mu\left(U_{1}\right)$ and by Condition $2 \mu(P)=\mu\left(P_{1}\right)$ and $\mu(U)$ $=\mu\left(U_{1}\right)$. Thus $N_{1}=U_{1}-U$ and $N_{2}=P_{1}-P$ are generalized nilpotents. Now

$$
T=U P=\left(U+N_{1}\right)\left(P+N_{2}\right)=U P+N_{1} P+U N_{2}+N_{1} N_{2}
$$

or

$$
-P N_{1}=\left(U+N_{1}\right) N_{2}
$$

hence

$$
\begin{aligned}
N_{2} & =-\left(U+N_{1}\right)^{-1} N_{1} P \\
& =-\left(\sum_{n=0}^{\infty}(-1)^{n}\left(U^{-1}\right)^{n+1} N_{1}^{n}\right) N_{1} P=\sum_{n=0}^{\infty}\left(-U^{-1} N_{1}\right)^{n+1} P .
\end{aligned}
$$

In order to apply these theorems we need the following result. 
Theorem 3. A spectral operator $T$ is a scalar operator whose spectrum lies on the unit circle if and only if: $T^{-1}$ is a bounded everywhere defined operator, and there exists a constant $M$ such that

$$
\left|T^{n}\right| \leqq M \quad n= \pm 1, \pm 2, \cdots .
$$

Proof. If $T=\int_{|\lambda|=1} \lambda E(d \lambda)$ then

$$
\left|T^{n}\right|=\left|\int_{|\lambda|=1} \lambda^{n} E(d \lambda)\right| \leqq 4 \sup \{|E(\alpha)| \mid \alpha \text { a Borel set }\} \text {, }
$$

by [1], p. 341. Conversely assume that $\left|T^{n}\right| \leqq M n= \pm 1, \pm 2, \cdots$ then

$$
R(\lambda ; T)= \begin{cases}\sum_{n=0}^{\infty} \frac{T^{n}}{\lambda^{n+1}}, & |\lambda|>1 \\ -\sum_{n=0}^{\infty} \lambda^{n}\left(T^{-1}\right)^{n+1}, & |\lambda|<1\end{cases}
$$

because that two series converge. Thus $\sigma(T) \subset\{\lambda|| \lambda \mid=1\}$ and $|R(\lambda ; T)|$ $\leqq M /|1-| \lambda||$ if $|\lambda| \neq 1$. By Lemma 3.16 of [2] if $T=S+N$, where $S$ is scalar and $N$ is a generalized nilpotent, then $N^{2}=0$. Hence

$$
T^{n}=S^{n}+n N S^{n-1} \text {. }
$$

Therefore $n N=\left(T^{n}-S^{n}\right) S^{-(n-1)}$.

Thus $n N$ is a bounded sequence of operators and therefore $N=0$.

LemMA 3. Let $S_{1}$ and $S_{2}$ be two commuting scalar operators with real spectra, if $S_{1}+S_{2}$ is spectral then it is scalar.

Proof. Let $S_{1}+S_{2}=S+N$ where $S$ is scalar and $N$ is a generalized nilpotent. By Theorem 3 the operator $e^{i(S+N)}=e^{i S_{1}} \cdot e^{i S_{2}}$ is a scalar operator, but

$$
e^{i(S+N)}=e^{i S} e^{i N}=e^{i S}+i N e^{i S} \sum_{n=1}^{\infty} \frac{(i N)^{n-1}}{n !},
$$

hence

$$
i N e^{i S} \sum_{n=1}^{\infty} \frac{(i N)^{n-1}}{n !}=0
$$

but the operator $i e^{i S} \sum_{n=1}^{\infty} \frac{(i N)^{n-1}}{n !}$ possesses an inverse and thus $N=0$.

THEOREM 4. Let $S_{1}$ and $S_{2}$ be two commuting scalar operators, if $S_{1}+S_{2}$ is spectral then

1. $S_{1}+S_{2}$ is a scalar operator. 
2. The real (imaginary) part of $S_{1}+S_{2}$ is the sum of the real (imaginary) parts of $S_{1}$ and $S_{2}$.

Proof. Let $S_{1}, S_{2}$ and $S_{1}+S_{2}$ be decomposed into real and imaginary parts as in Theorem 1 . Then

$$
S_{1}=R_{1}+i J_{1}, \quad S_{2}=R_{2}+i J_{2}, \quad S_{1}+S_{2}=R+i J
$$

where $R_{1}, J_{1}, R_{2}, J_{2}$ and $R$ are scalar operators, while $J$ is spectral, and would be scalar if and only if $S_{1}+S_{2}$ is a scalar operator. The operators $R_{1}, J_{1}, R_{2}, J_{2}$ commute and thus by the Gelfand theory [4] $R_{1}+R_{2}$ and $J_{1}+J_{2}$ have real spectra. By Theorem $1 R_{1}+R_{2}=R+M$ and $J_{1}+J_{1}=J$ $+i M$, where $M$ is a generalized nilpotent. By Lemma 3 the operator $R_{1}+R_{2}$ is a scalar operator, but $R$ is scalar too, thus by Theorem 8 of [1] $M=0$. Now $J_{1}+J_{2}=J$ which is a spectral operator and, again, by Lemma $3, J$ is scalar. Thus $S_{1}+S_{2}$ is scalar and $R_{1}+R_{2}=R, J_{1}+J_{2}=J$.

THEOREM 5. Let $S_{1}$ and $S_{2}$ be two commuting scalar operators. If $S_{1} S_{2}$ is spectral then

1. $S_{1} S_{2}$ is a scalar operator.

2. The absolute value (argument) of $S_{1} S_{2}$ is the product of the absolute values (arguments) of $S_{1}$ and $S_{2}$.

Proof. Let $S_{1}, S_{2}$ and $S_{1} S_{2}$ be decomposed as in Theorem 2 .

$$
S_{1}=P_{1} U_{1}, \quad S_{2}=P_{2} U_{2} \quad \mathrm{~S}_{1} S_{2}=P U .
$$

The operators $U_{1}, U_{2}, U, P_{1}$ and $P_{2}$ are scalar, and $P$ is a spectral operator, which is scalar if and only if $S_{1} S_{2}$ is scalar. Using commutativity of the operators in question and Theorem 2 we derive that

$$
P_{1} P_{2}=P+N_{2}, \quad U_{1} U_{2}=U+N_{1},
$$

where $N_{1}$ and $N_{2}$ are generalized nilpotents and $N_{2}=\sum_{n=0}^{\infty}\left(-N_{1} U^{-1}\right)^{n+1} P$. By Theorem 3, $N_{1}=0$ and hence $N_{2}=0$ too, which proves the second assertion. In order to complete the proof it remains to show that $P_{1} P_{2}$ is scalar. Now $P$ is spectral, let $P=P_{1} P_{2}=S+M$ where $S$ is scalar and $M$ a generalized nilpotent. Let $E(\cdot)$ and $F(\cdot)$ be the resolutions of the identity of $P_{1}$ and $P_{2}$ respectively. Denote $E\left\{\lambda \mid \lambda>\varepsilon_{1}\right\}=E_{\mathrm{\varepsilon}_{1}}$ and $F\{\lambda \mid \lambda$ $\left.>\varepsilon_{2}\right\}=F_{\varepsilon_{2}}$, then the spectrum of $E_{\varepsilon_{1}} P_{1} F_{\varepsilon_{2}} P_{2}=S E_{\varepsilon_{1}} F_{\varepsilon_{2}}+M E_{\varepsilon_{1}} F_{\varepsilon_{2}}$ on $E_{\varepsilon_{1}} F_{\varepsilon_{2}} X$ is contained in the set $\left\{\lambda \mid \lambda \geqq \varepsilon_{1} \varepsilon_{2}\right\}$ by the Gelfand theory. The operator $\log \left(E_{\varepsilon_{1}} P_{1} E_{\varepsilon_{2}} P_{2}\right)$ is thus well defined and it is not difficult to show that it is equal to $\log \left(E_{\varepsilon_{1}} P_{1}\right)+\log \left(E_{\varepsilon_{2}} P_{2}\right)$. This sum is spectral by [1], p. 340, and by Theorem 4 it is scalar. Thus $E_{\varepsilon_{1}} P_{1} F_{\varepsilon_{2}} P_{2}$ is scalar and therefore $M E_{\varepsilon_{1}} F_{\varepsilon_{2}}=0$. By countable additivity $M E_{0} F_{0}=0$ but $P_{1} E_{0}=P_{1}$ and $P_{2} F_{0}$ $=P_{2}$. Thus 


$$
P_{1} P_{2}=P_{1} E_{0} P_{2} F_{0}=S E_{0} F_{0}+M E_{0} F_{0}=S E_{0} F_{0},
$$

but $P_{1} P_{2}=S+M$, hence $S+M=S E_{0} F_{0}$, therefore $S=S E_{0} F_{0}$ and $M=0$ by Theorem 8 of [1]. Hence $P_{1} P_{2}=S$ is a scalar operator.

REMARK. From Theorems 4 and 5 it follows that the sum or product of two commuting spectral operators is spectral, if and only if, the sum or product of their scalar parts is scalar.

A decomposition of a non-spectral operator $A$ into real and imaginary parts is possible in some cases.

THEOREM 6. Let $A$ be an operator and $\sigma(A) \subset K$ where $K$ satisfies

1. There exists a function $f$ which is analytic and one-to-one in a neighborhood of $K$.

2. The image of $K$ is a subset of the unit circle.

3. The inverse function of $f$ exists and is analytic in a neighborhood of the unit circle, let us denote this function by $g$.

4. $g(\bar{z})=\overline{g(z)}$ if $|z|=1$.

Then $A=A_{1}+i A_{2}$ where $\sigma\left(A_{1}\right)$ and $\sigma\left(A_{2}\right)$ are sets of real numbers and $A_{1} A_{2}=A_{2} A_{1}$. If $A=B_{1}+i B_{2}$ where $B_{1}$ and $B_{2}$ satisfy the same conditions then $B_{1}=A_{1}+N$ and $B_{2}=A_{2}+i N$ and $N$ is a generalized nilpotent.

Proof. Let $\varphi(z)=g(1 / f(z))$ then $\varphi$ is analytic in a neighborhood of $K$ and for $z \in K, \varphi(z)=\bar{z}$. Define

$$
A_{1}=\frac{A+\varphi(A)}{2} \text { and } A_{2}=\frac{A-\varphi(A)}{2 i} .
$$

If $\mathfrak{A}$ is the full algebra generated by $A$ and $\mu \in \Delta_{\mathfrak{A}}$,

$$
\mu\left(A_{1}\right)=\frac{\mu(A)+\varphi(\mu(A))}{2}
$$

is the real part of $\mu(A)$, and $\mu\left(A_{2}\right)$ is the imaginary part of $\mu(A)$. Thus the first part of the theorem is proved. The second part is proved as in Theorem 1.

We conclude this section by a study of roots of operators. The operator $B$ is said to be an $n$th root of $A$ if $B^{n}=A$. The operators $A$ and $B$ commute $A B=B A=B^{n+1}$. Let $\mathfrak{A}$ be the full algebra generated by $B$. If $\mu \in \Delta_{\mathfrak{A}}$ then $\mu(B)^{n}=\mu(A)$ thus

$$
\sigma(B) \subset(\sigma(A))^{1 / n}
$$

Thus if $B^{n}=I$ then $\sigma(B) \subset\left\{\lambda \mid \lambda^{n}=1\right\}$ and hence is a finite set. By Theorem VII. 3.20 of [3], $B$ is spectral and by Theorem $3, B$ is a scalar operator. Thus 


$$
B=\sum_{k=0}^{n-1} e^{2 k \pi i / n} E_{k} \quad \text { where } E_{k}^{2}=E_{k}, E_{k} E_{j}=0
$$

if $k \neq j$, and $\sum_{k=0}^{n-1} E_{k}=I$.

THEOREM 7. Let $S$ be a scalar operator with real spectrum whose resolution of the identity is $E(\cdot)$. Let $S_{1}=\int \lambda^{1 / n} E(d \lambda)$ where $\arg \lambda^{1 / n}$ $=(\arg \lambda) / n$. If $S_{2}$ satisfies $S_{2}^{n}=S$, then $\sigma\left(S_{2}\right) \subset(\sigma(S))^{1 / n}$, and if $\sigma\left(S_{2}\right) \subset\left\{\lambda^{1 / n} \mid \lambda\right.$ $\in \sigma(S)$ and $\left.\arg \lambda^{1 / n}=(\arg \lambda) / n\right\}$ then

$$
S_{2}=S_{1}+N \text { and } N=N E(\langle 0\rangle) \text { and } N^{n}=0 \text {. }
$$

Proof. The operators $S_{1}$ and $S_{2}$ commute by [1] p. 329. Let $\mathfrak{A}$ be the full algebra generated by them. If $\mu \in \Delta_{\mathfrak{H}}$ then $\mu\left(S_{1}\right)=\mu\left(S_{2}\right)$ and thus $S_{2}-S_{1}=N$ is a generalized nilpotent. Now

$$
S=S_{2}^{n}=S_{1}^{n}+n N S_{1}^{n-1}+\frac{n(n-1)}{2} N^{2} S_{1}^{n-2}+\cdots+N^{n}=S_{1}^{n}
$$

therefore

$$
N\left(n S_{1}^{n-1}+\frac{n(n-1)}{2} N S_{1}^{n-2}+\cdots+N^{n-1}\right)=0
$$

but by Corollary 4 of Theorem 1 , Section $3, N S_{1}^{n-1}=0$. Thus by Theorem 2 of $\S 3, N=N E(\langle 0\rangle)$, but then $N S_{1}^{q}=0$ for every integer $q$. Instead of (1) we have, therefore,

$$
S=S_{1}^{n}+N^{n} \quad \text { or } \quad N^{n}=0
$$

which completes the proof.

\section{REFERENCES}

1. N. Dunford, Speclral operators. Pacific J. Math. 4 (1954), 321-354.

2. Spectral theory. II Resolutions of the identity. Pacific J. Math. 2 (1952), 559-614.

3. _ , and J. Schwartz, Linear operators. Interscience Pub., New York.

4. I. M. Gelfand, Normierte Ringe. Mat Sb. N. S. 9 (51), (1941), 3-24.

5. P. R. Halmos, Introduction to Hilbert space and the theory of spectral multiplicity. Chelsea, New York, 1951.

6. A. C. Zaanen, Linear analysis. P. Noordhoff, Groningen, and Interscience Pub., New York, 1953.

YALE UNIVERSITY 



\section{PACIFIC JOURNAL OF MATHEMATICS}

\section{EDITORS}

David Gilbarg

Stanford University

Stanford, California

R. A. Beaumont

University of Washington

Seattle 5 , Washington
A. L. Whiteman

University of Southern California Los Angeles 7, California

E. G. Straus

University of California

Los Angeles 24, California

\section{ASSOCIATE EDITORS}
E. F. BECKENBACH
C. E. BURGESS
M. HALL
E. HEWITT
A. HORN
V. GANAPATHY IYER
R. D. JAMES
M. S. KNEBELMAN

L. NACHBIN

I. NIVEN

T. G. OSTROM

H. L. ROYDEN
M. M. SCHIFFEI

G. SZEKERES

F. WOLF

K. YOSIDA

\section{SUPPORTING INSTITUTIONS}

UNIVERSITY OF BRITISH COLUMBIA

CALIFORNIA INSTITUTE OF TECHNOLOGY

UNIVERSITY OF CALIFORNIA

MONTANA STATE UNIVERSITY

UNIVERSITY OF NEVADA

OREGON STATE COLLEGE

UNIVERSITY OF OREGON

OSAKA UNIVERSITY

UNIVERSITY OF SOUTHERN CALIFORNIA
STANFORD UNIVERSITY

UNIVERSITY OF TOKYO

UNIVERSITY OF UTAH

WASHINGTON STATE COLLEGE

UNIVERSITY OF WASHINGTON

AMERICAN MATHEMATICAL SOCIETY

CALIFORNIA RESEARCH CORPORATION

HUGHES AIRCRAFT COMPANY

THE RAMO-WOOLDRIDGE CORPORATION

Printed in Japan by Kokusai Bunken Insatsusha

(International Academic Printing Co., Ltd.), Tokyo, Japan 


\section{Pacific Journal of Mathematics}

\section{Vol. 8, No. 1 \\ March, 1958}

Shimshon A. Amitsur, Commutative linear differential operators ......... 1

Masahiko Atsuji, Uniform continuity of continuous functions of metric

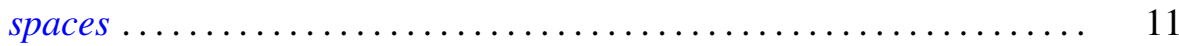

S. P. Avann, A numerical condition for modularity of a lattice . . . . . . . . . 17

Raymond G. D. Ayoub, A mean value theorem for quadratic fields........ 23

Errett Albert Bishop, Subalgebras of functions on a Riemann surface ..... . 29

Shaul Foguel, The relations between a spectral operator and its scalar

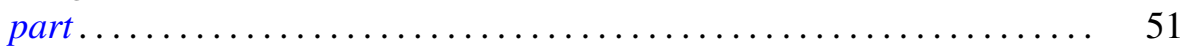

John Rolfe Isbell, Euclidean and weak uniformities ................. 67

Samuel Karlin and James L. McGregor, Many server queueing processes with Poisson input and exponential service times .............. 87

Paul Joseph Kelly and Ernst Gabor Straus, Curvature in Hilbert geometries....................................... 119

John W. Lamperti, Stationary measures for certain stochastic processes . . . 127

Richard Scott Pierce, Distributivity and the normal completion of Boolean

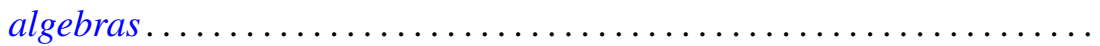

F. M. Ragab, Transcendental addition theorems for the hypergeometric

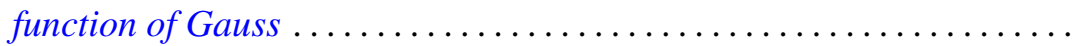

William T. Reid, Principal solutions of non-oscillatory self-adjoint linear differential systems ................................ 147

Maurice Sion, On general minimax theorems .................... 171

Chien Wenjen, On semi-normed ${ }^{*}$-algebras .................... 177 